\title{
The quantum Hall effect: spin-charge locking
}

\author{
Hasan Abu Kassim, Ithnin Abdul Jalil, Norhasliza Yusof and Keshav N. Shrivastava*
}

Department of Physics, Universiti Malaysa, 50603 Kuala Lumpur, Malaysia

*To whom correspondence should be addressed. E-mail: keshav@um.edu.my

Received 27 March 2006

http://dx.doi.org/10.11113/mjfas.v2n1-2.15

\begin{abstract}
In two-dimensional electron gas when a large magnetic field is applied in one direction and an electric field perpendicular to it, there is a current in a direction perpendicular to both. This current is called the Hall effect. It remained without quantization until 1980 when it was found that the quantization leads to correct measurement of $h / \mathrm{e}^{2}$. Therefore the quantized Hall effect was further studied at high magnetic fields where fractional quantization was found. The fractional charge can arise from the "incompressibility" in the flux quantization. Laughlin wrote a wave function, the excitations of which are fractionally charged quasiparticles. This wave function comes in competition with charge density waves but for a few fractions it does give the ground state. If "incompressibility" is not considered and it is allowed to be compressible, the fractional charge can arise from the angular momentum which appears in the Bohr magneton in the form of $\mathrm{g}$ values. Usually the positive spin is considered but we consider both the positive as well as the negative values so that there is a spin-charge coupling. The values thus calculated for the fractional charge agree with the experimental data on the quantum Hall effect. We have followed this subject for a long time and hence have reviewed the subject. There are several interesting concepts which we learn from this subject. The concept of the Hall effect is quite clear particularly when combined with the flux quantization. We learn about the Landau levels and hence the boson character of electrons in two dimensions. We learn that charge becomes a vector quantity and there is spincharge coupling.
\end{abstract}

| Hall effect | spin-charge coupling | GaAs |

\section{Introduction}

In a semiconductor, when an electric field is applied along the $\mathrm{x}$ direction and a magnetic field is applied along the $\mathrm{z}$ direction, then a current appears in the $\mathrm{y}$ direction. In 1878 Edwin Hall measured the voltage along the $\mathrm{y}$ direction and found that it is linearly proportional to the applied field along the $\mathrm{z}$ direction. Hence the appearence of voltage along the y direction is called the Hall effect. In 1980, it was found[1] that the Hall resistivity does not quite follow the linear dependence on magnetic field but there are small plateaus at certain values. The value of resistivity at the plateau has the correct value of $\mathrm{h} / \mathrm{e}^{2}$. This phenomenon is called the "quantum Hall effect" and it is obviously useful for obtaining the correct values of the fundamental constants. When the measurements were extended to higher magnetic fields, it was found that there is a quantization at $(1 / 3) \mathrm{e}$. It was thought that the physical origin of the fractional values must be very different from that of integer quantized resistivity and hence 
it was named, "fractional quantum Hall effect" [2]. In 1983, there is no effort to find the orgin of the quantization particularly at the fractional values but Laughlin [3] used incompressibility in the flux quantization to find a wave function which gives quasiparticles of fractional charges. It was found that the charge density wave state has higher energy so the fractionally charged state becomes the ground state. For theoretical physics it is sufficient to prove that there is a ground state the excitations of which are fractionally charged. However, the wave functions are not useful for calculating physical properties of solids such as resistivity. By fixing the area in the flux quantization, it is possible to make the fractional charge but this is not what happens in the real experiment. There is no mechanism to hold the area to maintain the fractional charge and if area is relaxed the fractional charge disappears. The spin of the electron also does not play any particular role in holding the area incompressible. In 1985, Shrivastava[4] predicted the fractional charges correctly by inventing certain spin symmetries. In this theory, fixing the spin, fixes the charge so that there is spin-charge locking. The symmetries of the predicted charges as well as their magnitudes are in agreement with the experiments [5,6]. When the spin reverses, the charge also changes so that there is an effect of Kramer's time reversal on the change in the charge of the quasiparticles. Therefore, there is a particle-hole symmetry [5]. Usually, the spin of the electron is a positive number but we use also the negative spin [7] so that in going from one charge to another, momentum is conserved. In this way we are able to understand all of the data on the quantum Hall effect [8-14].

In this article, we give the interpretation of the data on quantum Hall effect and describe some new spin properties which lead to fractional charge. The usual spin s is to be replaced by $\left(\begin{array}{cc}s & 0 \\ 0 & -S\end{array}\right)$,

which produces fractional charges by means of the $\mathrm{z}$ component of the spin and the Bohr magneton.

\section{The Hall Effect}

In a semiconductor with electric field along $\mathrm{x}$ direction and the magnetic field along $\mathrm{z}$ direction, the force acting on the electron is, $-\mathrm{e} \mathrm{v} \times \mathrm{B}$. The force on the electron $\mathrm{m} \mathrm{dv} / \mathrm{dt}=\hbar \mathrm{dk} / \mathrm{dt}$ involves second derivative of distance with respect to time so that there is a change in the force during the time $\mathrm{dt}$ and the Galilean transformation introduces the velocity dependent force. We apply the Newton's law of motion so that, $\hbar d \mathbf{k} / \mathrm{dt}=-\mathrm{e}[\mathbf{E}+(1 / \mathrm{c}) \mathbf{v} \times \mathbf{B}]$. We leave out the relativistic transformation of mass so that when $B=0$, hdk=-eEdt. We introduce a finite life time effect by integrating from 0 to $\tau$ during which the field is applied. In this time interval, $\mathbf{k}$ changes from $\mathbf{k}(0)$ to $\mathbf{k}(\tau)$ which we can write as if the electron acquires the velocity, $\mathbf{v}$ so that the momentum can be integrated to, $\mathrm{mv}=\hbar \mathrm{dk}=-\mathrm{eE} \tau$. Therefore, the velocity of the electron upon the application of an electric field becomes, $\mathrm{v}=-\mathrm{eE} \tau / \mathrm{m}$. We assume that the mass of the free electron is changed to $\mathrm{m}^{*}$ because of a periodic potential in a crystal. Therefore, the force on the electron may be written as, $\mathrm{m}^{*}(\mathrm{dv} / \mathrm{dt}+(1 / \tau) \mathrm{v})=\mathrm{e}(\mathrm{E}+(1 / \mathrm{c}) \mathrm{v} \times \mathrm{H})$. The relaxation time of the electrons which have now become the charge carriers is related to the mean free path, $\Lambda=|v| \tau$, where $|v|$ is the magnitude of the mean particle velocity. We take the steady state with $\mathrm{dv} / \mathrm{dt}=0$ so that the carrier velocity in terms of components becomes, $v_{x}=t_{1} E_{x}+h v_{y} ; v_{y}=t_{1} E_{y}-h v_{x} ; v_{z}=t_{1} E_{z}$ where we have abbreviated the coefficients as follows, $\mathrm{t}_{1}=\mathrm{e} \tau / \mathrm{m}^{*}$ and $\mathrm{h}=\mathrm{t}_{1} \mathrm{H} / \tau=\mathrm{eH} \tau / \mathrm{m}^{*} \mathrm{c}=-\omega_{\mathrm{c}} \tau$.. Using the definition of the current $\mathrm{j}=$ nev, one can write down the conductivity as a $3 \times 3$ matrix. Taking $\mathrm{j}_{\mathrm{y}}$ and $\mathrm{E}_{\mathrm{x}}$ the $\mathrm{xy}$ component of the conductivity is found to be $\sigma_{\mathrm{xy}}=\mathrm{nec} / \mathrm{H}$. The Hall resistivity is thus linearly proportional to the applied magnetic field. This is the Hall effect.

\section{Flux Quantization and quantum Hall Effect}

The magnetic field is usually a continuous variable. We produce the magnetic field by means of making a coil and passing d.c. currents through it. In the case of superconductors, the magnetic field is zero due to pairing of electrons. However, in a cup shaped sample, when field a applied it got quantized in a small area near the edge. 
So when effort is made to push a field in a ring of a superconductor, quantized field is found. The magnetic field $\mathrm{B}$ became quantized according to $\mathrm{B} . \mathrm{A}=\mathrm{nhc} / 2 \mathrm{e}$. Here $\mathrm{A}$ is the area in which field got quantized, $\mathrm{n}$ is an integer, $\mathrm{n}=1,2,3, \ldots$, etc., $\mathrm{h}$ is the Planck's constant, $\mathrm{c}$ is the velocity of light and $\mathrm{e}$ is the charge of the electron. The factor of 2 shows that there are pairs of electrons so that the unit of charge is 2e instead of the usual e. When this quantization is substituted in the Hall effect formula, the resistivity got quantized as, $\rho_{x y}=h / i^{2}$ where $i=1,2,3, \ldots$, This is called the quantum Hall effect. An effort was made to repeat this experiment at higher magnetic fields compared with that at $i=1$. When the field was increased to thrice this value, there occurred a plateau in the resistivity at $\rho_{x y}=h /(1 / 3) e^{2}$,i.e., at $i=1 / 3$ so that the charge of the quasiparticle is (1/3)e. This is called the fractional quantum Hall effect.

This means that the field is quantized in the superconductors in the units of hc/2e and in normal semiconductors in units of hc/e. The integer charges are easy to understand because it is in units of 1e but the fractions are more difficult to understand theoretically unless there is some new effect associated with electrons in a magnetic field. We have to discover this behavior of electrons at high magnetic fields. It will turn out that spin plays an important role and the usual flux quantization itself depends on spin.

\section{Laughlin's wave function}

Laughlin used the flux quantization to obtain the fractional charge from the relation B.A=nhc/2e. The quantized field becomes, $\mathrm{B}=\mathrm{nhc} / 2 \mathrm{Ae}$. So that fixing the area and the charge product as a constant, $3 \mathrm{~A}$ and e/3 will keep the formula unchanged. We can fix the area at $3 \mathrm{~A}$ and introduce "incompressibility" so that the charge becomes e/3 and this is the modern theory of fractional charges by squeezing the area. Is this what happens in the experiment? That we have to find out. Let us see the Laughlin's approach. First of all Laughlin wrote the Hamiltonian for electrons in two dimensions because that will conform to the experimental configuration. The electrons in the xy plane form Landau levels so the Hamiltonian should be,

$$
\mathrm{H}=\Sigma_{\mathrm{j}=1}{ }^{\mathrm{N}}\left\{\left|(\mathrm{h} / \mathrm{i}) \nabla_{\mathrm{j}}-(\mathrm{e} / \mathrm{c}) \mathrm{A}_{\mathrm{j}}\right|^{2}+\mathrm{V}\left(\mathrm{z}_{\mathrm{j}}\right)\right\}+\Sigma_{\mathrm{j}>\mathrm{k}} \mathrm{e}^{2} /\left|\mathrm{z}_{\mathrm{j}}-\mathrm{Z}_{\mathrm{k}}\right|
$$

Where $\mathrm{N}$ is the number of particles and $\mathrm{V}$ is the potential due to uniform background of positive charges. The only wave function composed of states in the lowest Landau level which have angular momentum $\mathrm{m}$ and orbit about the centre of mass are of the form,

$$
\psi=\left(\mathrm{z}_{1}-\mathrm{z}_{2}\right)^{\mathrm{m}}\left(\mathrm{z}_{1}+\mathrm{z}_{2}\right)^{\mathrm{n}} \exp \left[-\frac{1}{4}\left(\left|\mathrm{z}_{1}\right|^{2}+\left|\mathrm{z}_{2}\right|^{2}\right)\right] \text {. }
$$

The $|\psi|^{2}$ describes a system of uniform density, $(1 / \mathrm{m})(1 / \mathrm{A})$, which is the charge per unit area. Therefore, $\mathrm{m}=3$ corresponds to a charge of e/3. Laughlin examined the energy minima and showed that $1 / 3$ energy minima is deeper than that of the charge density waves. The number $\mathrm{m}$ can vary, so when $\mathrm{m}=1 / 7$ the energy of the fractionally charged state crosses that of the charge density waves.

\section{Composite Fermions}

It was suggested that over and above the flux associated with the motion of the electron, additional flux quanta if attached to the electron, may explain some of the properties of the quantum Hall effect. In particular, the fractions observed and some of the symmetries can be obtained if the number of flux quanta attached to the electron is even. For example, if two flux quanta are attached to one electron it is called a CF model. Similarly, the square of that number such as $2 / 3$ in case of $1 / 3$, will have to be a boson and these flux quanta attached to the electron form composite bosons. These models are unphysical and hence are better discarded [10] when interpretation of the real experiments is concerned. 


\section{Angular Momentum Theory}

Usually the free electrons are in the $l=0$ state so that the Millikan oil drop method or electron gas models give a single value of the charge of the electron. In these experiments $l=0$ is the root cause of observing a single value for the electron charge. We [4-7] consider (a) the finite value of $l$ and more spin symmetries than are usually taken into account. We allow two levels for $\mathrm{s}=1 / 2$ and two more levels for spin $=-1 / 2$ so that there are four levels for spin $1 / 2$. These levels are not superimposed on each other because there are two separate $g$ values, one for spin $+1 / 2$ and the other for spin $-1 / 2$. We consider the spin as well as the orbital motion so that,

$$
\mathrm{g}_{j} j=g_{s} \mathrm{~s}+g_{l} l=\frac{1}{2}\left(\mathrm{~g}_{l}+\mathrm{g}_{\mathrm{s}}\right) j+\frac{1}{2}\left(\mathrm{~g}_{l}-\mathrm{g}_{\mathrm{s}}\right)(l-\mathrm{s}) .
$$

Multiplying both sides of the above equation by $\boldsymbol{j}=\boldsymbol{l}+\boldsymbol{s}$ and taking eigen values, we find,

$$
\mathrm{g}_{\mathrm{j}} \mathrm{j}(\mathrm{j}+1)=\frac{1}{2}\left(\mathrm{~g}_{1}+\mathrm{g}_{\mathrm{s}}\right) \mathrm{j}(\mathrm{j}+1)+\frac{1}{2}\left(\mathrm{~g}_{1}-\mathrm{g}_{\mathrm{s}}\right)[l(l+1)-\mathrm{s}(\mathrm{s}+1)] .
$$

Which upon substituting $\mathrm{s}=1 / 2$ gives $\mathrm{j}=l \pm(1 / 2)$ for which,

$$
\mathrm{g}_{\mathrm{j}}=\mathrm{g}_{1} \pm \frac{g_{s}-g_{l}}{2 l+1} .
$$

For $\mathrm{g}_{\mathrm{s}}=2, \mathrm{~g}_{\mathrm{l}}=1$ we find,

$$
\mathrm{g}_{ \pm}=1 \pm \frac{1}{2 l+1} \text {. }
$$

Usually the treatment of cyclotron resonance is for free electrons but we need to introduce the concept of $g$ values in the cyclotron resonance also. The usual expression is, $\omega=\mathrm{eB} / \mathrm{mc}$. Corresponding to this frequency, the voltage along y direction is $\hbar \omega=\mathrm{eV}_{\mathrm{y}}$. From the last two expressions, $\hbar \mathrm{eB} / \mathrm{mc}=\mathrm{eV}_{\mathrm{y}}$. We multiply the above by e/h from both sides so that,

$$
\frac{e^{2} B}{2 \pi m c}=\frac{e^{2}}{h} V_{y}
$$

which describes the current in the $\mathrm{x}$ direction so that it is proved that,

$\rho_{x y}=\frac{h}{e^{2}}$ which is the same as that required for the quantum Hall effect. We take into account the gyromagnetic ratio so that (7) may be written as,

$$
\mathrm{I}_{\mathrm{x}}=\frac{1}{2} \mathrm{~g} \frac{e^{2} B}{2 \pi m c}=\frac{1}{2} g \frac{e^{2} V_{y}}{h} .
$$

For $l=0, \mathrm{~g}=2$,

$$
\mathrm{I}_{\mathrm{x}}=\frac{e^{2}}{h} V_{y}
$$

which describes the quantized current correctly for $v=1$. From (8) $v=\frac{1}{2} g_{ \pm}$which gives the filling factor, one value for + sign and the other for - sign in (6). We have thus introduced three different $g$ values, the usual $g$ value as well as $g_{ \pm}$. For $l=0$, we obtain $(1 / 2) g_{+}=1$ and $(1 / 2) g_{-}=0$, for $l=1$, we get $(1 / 2) g_{+}=2 / 3$ and $(1 / 2) g_{-}=1 / 3$. These values of $v=(1 / 2) g_{ \pm}$are given in Table 1 from the theory of Shrivastava[4] which is also described in refs. 8 and 9. The predicted values are the same as those measured by Stormer[11] as given in Fig.1. The effective charge is determined by the modification of the cyclotron frequency, $\hbar \omega_{c}=g \mu_{B} B$ as, $\mathrm{e}_{\mathrm{eff}}=(1 / 2) \mathrm{g}_{ \pm} \mathrm{e}=v \mathrm{e}$. 


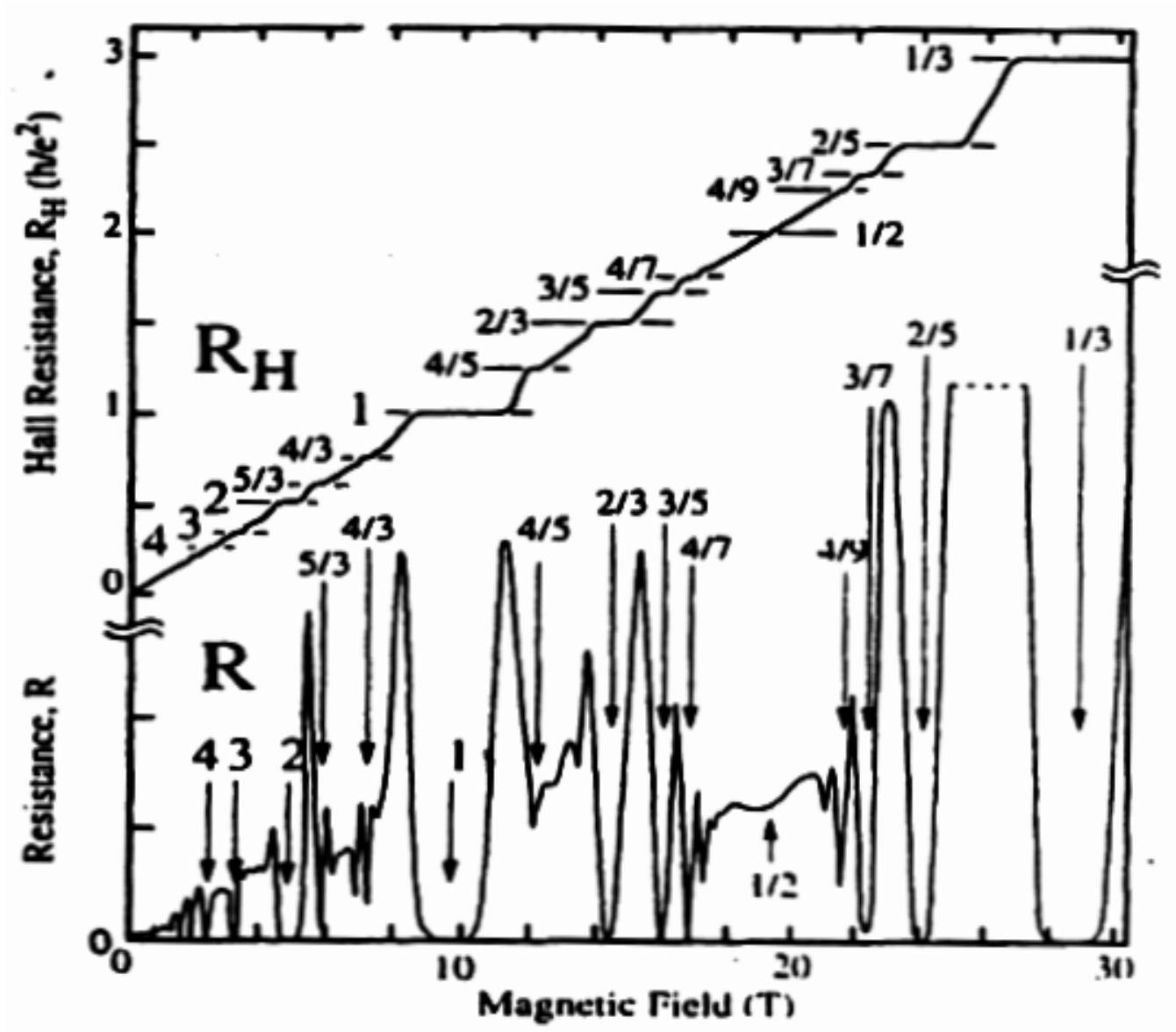

Fig.1. The experimentally measured (by Stormer) Hall effect resistivity showing same fractions as those predicted by us.

\section{Supersymmetry and Tower of States}

In the case of supersymmetry, the Hamiltonian is written in terms of two components which are given in terms of $\mathrm{L}$ and $\mathrm{L}^{\dagger}$,

$$
\begin{aligned}
& \mathrm{H}=\left(\begin{array}{cc}
H_{1} & 0 \\
0 & H_{2}
\end{array}\right) ; \\
& H_{1}=\mathrm{L}^{\dagger} \mathrm{L} \text { and } H_{2}=\mathrm{LL}^{\dagger}
\end{aligned}
$$

which define the charge in terms of $2 \times 2$ matrices, 


$$
\mathrm{Q}=\left(\begin{array}{ll}
0 & 0 \\
L & 0
\end{array}\right) \text { and } \mathrm{Q}_{+}=\left(\begin{array}{cc}
0 & \mathrm{~L} \dagger \\
0 & 0
\end{array}\right)
$$

which obey the commutators,

$$
\left[\mathrm{H}, \mathrm{Q}_{ \pm}\right]=\left\{\mathrm{Q}_{ \pm}, \mathrm{Q}_{ \pm}\right\}=0 ;\left\{\mathrm{Q}_{-}, \mathrm{Q}_{+}\right\}=\mathrm{H}
$$

where $\mathrm{LH}_{1}=\mathrm{H}_{2} \mathrm{~L}$ and $\mathrm{L}$ is given by Darboux transformation [12]. We define $\kappa=-(\mathrm{j}+1 / 2)<0$ with $l^{\prime}=l+1$ for aligned and $\kappa=(j+1 / 2)>0, l^{\prime}=l-1$ unaligned, there are two towers for states, one for $\kappa<0$ and the other for $\kappa>0$. In our problem both signs of spin are taken into account so that the spin $\mathrm{s}$ is replaced by the matrix,

$$
\left(\begin{array}{cc}
s & 0 \\
0 & -s
\end{array}\right)
$$

and there are four levels for $\mathrm{s}_{\mathrm{z}}=1 / 2$ two for $\mathrm{g}_{+}$and two for $\mathrm{g}$. which describe the fractional charges. As we change the value of $l$ we get two towers of states, one for + sign of the spin and the other for - sign.

\section{Spin-Charge Locking}

The charge of the electron is a scalar number. If it is written as a matrix it may be just -e multiplied by a unit matrix. Even then it is independent of spin. If we invent an isospin for the electron's charge, then also it will be independent of the spin. However, in our problem changing the spin value changes the charge of the electron. If it is $1 / 3$ for the negative sign of the spin it is $2 / 3$ for the positive sign. So we assign a matrix, similar to the isospin for the charge. If the $\mathrm{z}$ component of the isospin aligns with the $\mathrm{z}$ component of the spin, there is spin-charge locking. In our theory, the charge in dimensionless units is given by,

$$
\mathrm{e}^{*}=\{l+(1 / 2) \pm \mathrm{s}\} /(2 l+1) .
$$

In the case of a superconductor the $\mathrm{s}_{\mathrm{x}}$ aligns with $\mathrm{e}_{\mathrm{x}}{ }_{\mathrm{x}}$ so that there is spin-charge locking [13]. Since the resistivity is strictly $\mathrm{h} / \mathrm{e}^{2}$ there must be spin-charge coupling. Let us look at the dot product of the spin with charge,

$$
\mathrm{e}^{*} . \mathrm{s}^{\prime}=\frac{1}{2 l+1}[l+(1 / 2) \pm \mathrm{s}] . \mathrm{s}^{\prime}=\frac{1}{2 l+1}\left[l \cdot \mathrm{s}^{\prime}+(1 / 2) \mathrm{s}^{\prime} \pm \mathrm{s}\right]
$$

in which the first term is the spin-orbit coupling but the two need not belong to one and the same particle. The second term is the ordinary spin divided by $2 l+1$ and the last term is the spin-spin interaction. This way of starting with the effective charge, thus produces entangled spin-orbit interactions. There is no doubt about the existence of this type of interactions and that they produce the fractional charges. As we have the spin-spin interaction, we can also have the charge-charge type interaction. This again produces the orbit-orbit interaction in which both the orbits need not belong to one and the same electron. Therefore, when we compare it with the Heisenberg interaction, we find that orbital ordering can be predicted. There is additional spin-spin interaction which also has the orbital angular momentum in the denominator. 
Table 1: The calculated values of fractional charges for various values of $l$ and $s= \pm 1 / 2$.

\begin{tabular}{|c|c|c|c|}
\hline S.No. & $l$ & $(1 / 2) \mathrm{g}=l /(2 l+1)$ & $(1 / 2) \mathrm{g}_{+}=(l+1) /(2 l+1)$ \\
\hline 1 & 0 & 0 & 1 \\
\hline 2 & 1 & $1 / 3$ & $2 / 3$ \\
\hline 3 & 2 & $2 / 5$ & $3 / 5$ \\
\hline 4 & 3 & $3 / 7$ & $4 / 7$ \\
\hline 5 & 4 & $4 / 9$ & $5 / 9$ \\
\hline 6 & 5 & $5 / 11$ & $6 / 11$ \\
\hline 7 & 6 & $6 / 13$ & $7 / 13$ \\
\hline 8 & 7 & $7 / 15$ & $8 / 15$ \\
\hline 9 & $\infty$ & $1 / 2$ & $1 / 2$ \\
\hline
\end{tabular}

\section{Conclusions}

The quantum Hall effect is predicted by using the flux quantization. The fractions of charges found in the experimental data on the quantum Hall effect are well predicted by using new spin symmetries. The usage of negative spin and 4 states for spin $1 / 2$ are characteristic features of this theory. There is spin-charge coupling. The change in spin is adjusted by a change in the orbital state. The charge of a particle changes upon changing spin. This means that the Coulomb orbits are different for different spins. There are spin-spin and spin-orbit interactions. The usual Landau levels occur at $\hbar \omega(n+1 / 2)$. This must be corrected to take into account the new effects to $(1 / 2) g_{ \pm} \hbar \omega$, so that the cyclotron frequency splits for the two signs as well as there are a whole series of charges as given in the Table 1 .

\section{Acknowledgements}

The authors acknowledge the support provided by the University of Malaya. 


\section{References}

[1] K. von Klitzing, G. Dorda and M. Pepper, Phys. Rev. Lett. 45 (1980) 494.

[2] D. C. Tsui, H. L. Stormer and A. C. Gossard, Phys. Rev. Lett. 48 (1982) 559.

[3] R. B. Laughlin, Phys. Rev. Lett, 50 (1983) 1395.

[4] K. N. Shrivastava, Phys. Lett. A 113 (1986) 435.

[5] K. N. Shrivastava, Mod. Phys. Lett. 13 (1999) 1087.

[6] K. N. Shrivastava, Mod. Phys. Lett. 14 (2000) 1009.

[7] K. N. Shrivastava, Phys. Lett. A 326 (2004) 469.

[8] K. N. Shrivastava, Introduction to quantum Hall effect, Nova Science Publishers, Inc. New York 2002.

[9] K. N. Shrivastava, Quantum Hall effect: Expressions, Nova Science Publishers, Inc. New York 2005.

[10] W. Pan, H. L. Stormer, D. C. Tsui et al Phys. Rev. Lett. 90 (2003) 016801.

[11] H. L. Stormer, Rev. Mod. Phys. 71 (1999) 875.

[12] A. Leviatan, Phys. Rev. Lett. 92 (2004) 202501.

[13] P. W. Anderson, Phys. Rev. Lett. 96 (2006) 017001. 